\title{
Neurosyphilis Presenting with Papillitis
}

\author{
Martin Edward Perry ${ }^{1}$,Sarah Cooper ${ }^{2}$, Shona Corry ${ }^{2}$ \\ ${ }^{1}$ Department of Rheumatology, Royal Alexandra Hospital, Paisley, UK \\ 2Undergraduate Medical School, University of Glasgow, Glasgow, UK
}

Received: 31/07/2017

Accepted: $19 / 08 / 2017$

Published: 05/09/2017

How to cite this article: Perry ME, Cooper S, Corry S. Neurosyphilis presenting with papillitis. EJCRIM 2017;4: doi:10.12890/2017_000718.

Conflicts of Interests: The Authors declare that there are no competing interests.

This article is licensed under a Commons Attribution Non-Commercial 4.0 License

\section{ABSTRACT}

Syphilis is one of the oldest described infectious diseases in the world and is caused by the spirochete bacterium Treponema pallidum ${ }^{[1]}$. Although now a rare disease, incidence is increasing with the number of diagnoses of the disease rising in England from 1688 to 2713 between 2003 and 2012 (a 61\% increase) ${ }^{[2]}$. Major outbreaks of syphilis have been documented in London, Manchester, Dublin, and Brighton particularly among men who have sex with men (MSM) ${ }^{[3]}$. Diagnosis remains difficult on account of multi-system symptoms, duration of the condition, and social stigma.

\section{LEARNING POINTS}

- Normal CSF pressure with indistinct optic disc margins suggests papillitis, not papilloedema

- Neurosyphilis can cause various ocular symptoms and is a cause of unexplained high ESR

- When treating with penicillin, the physician should be aware of a potential Jarish-Herzheimer reaction

\section{KEYWORDS}

Neurosyphilis, papillitis, Treponema pallidum

\section{CASE DESCRIPTION}

A 49-year-old man presented with a severe headache and progressive visual blurring. He was referred to general internal medicine from ophthalmology where he had undergone investigations for headache and increasingly blurred vision. Symptom duration was three to four weeks with sharp relentless pain in his head which he explained had been almost constant for nearly four weeks. The pain was at its most severe behind his eyes but radiated to the parietal and occipital regions of his head. The patient felt lethargic and generally unwell with episodes of sweats but reported no nausea or vomiting, denied any weight loss, and exhibited no speech disturbances or other obvious neurological deficit.

However, he volunteered that a rash had developed over the past 6 weeks across his arms and legs.

Prior to these symptoms, the patient was generally healthy and had no extensive past medical history but he had recently experienced some rectal bleeding along with a four-week history of diarrhoea and mucus for which he had undergone sigmoidoscopy which confirmed proctitis and he was awaiting an MRI of his pelvis to provide further clarification. He was not on any long-term medication but had recently been prescribed amoxicillin by his GP for a presumed chest infection.

On examination the patient looked well and had an obvious maculopapular rash across arms and legs sparing the hands. It was a non pruritic, florid, and erythematous. His neurological examination was normal, apart from papilloedema which was evident on fundoscopy, and a nystagmus which was present on the right side. Further slit lamp ophthalmological assessment confirmed bilateral papilloedema with 
haemorrhage but no relative afferent pupillary defect. His chest was clear and he had a regular pulse. His basic observations including blood pressure, heart rate, oxygen saturation, respiratory rate, and temperature were all normal.

Four days prior to admission the patient had a normal CT scan of his brain and was waiting for a brain MRI. Blood tests taken by the ophthalmologist also revealed an elevated erythrocyte sedimentation rate (ESR) at $120 \mathrm{~mm} / \mathrm{hr}$.

Further blood tests were ordered which showed no abnormalities in urea and electrolytes thyroid function or in lyme serology, rheumatoid factor, $\mathrm{C}$ reactive protein, ANA, ANCA, and serum ACE. However, he had mild anaemia (Hb 125g/l), reduced serum albumin (29), elevated alkaline phosphatise (350), and reduced complement C4. His viral serology was negative for HIV, hepatitis, herpes simplex, varicella, enterovirus, and parechovirus. ASO titre was modestly raised suggesting previous infection. Stool cultures were negative for cysts, ova, and parasites. A lumbar puncture was performed with a normal opening pressure of $15 \mathrm{mmHg}$. Cerebrospinal fluid (CSF) protein and glucose were both in the normal range and no organisms were seen at 48 hour culture. CSF oligoclonal bands were absent. However, CSF culture for the spirochete Treponema pallidum was highly positive. He was diagnosed with neurosyphilis.

Biopsy of the rash confirmed a prominent lymphohistiocytic minfiltrate within the papillary dermis, focally obscuring the dermo-epidermal junction. It was felt to be consistent with a drug eruption, possibly related to the recently used amoxicillin.

Following the positive finding of the spirochete Treponema pallidum the patient subsequently revealed that he had been engaging in unprotected anal intercourse with multiple male partners for the past 18 months, but claimed he had a negative syphilis serology 2 years ago. He was started on a course of procaine penicillin IV for 14 days.

\section{DISCUSSION}

As highlighted in this case, neurological symptoms during syphilis infection can best be diagnosed by examination and culture of the cerebrospinal fluid. The presence of blurred optic discs (Fig. 1) should indicate papillitis. This is suspected when the opening pressure of CSF is in the normal range and so this is not papilloedema. Papillitis has many causes as outlined in Table 1 which should be considered in the differential diagnosis. Treponema $p$. infection can also cause a variety of additional ophthalmological infections as outlined in Table $2^{[1]}$. Neurosyphilis develops in approximately $25-40 \%$ of patients with untreated syphilis ${ }^{[4]}$. Without treatment insidious development of symptoms can include personality change, psychosis delirium, and dementia. Physical change can include stroke, myelopathy, seizure, brainstem symptoms, and ocular abnormalities.

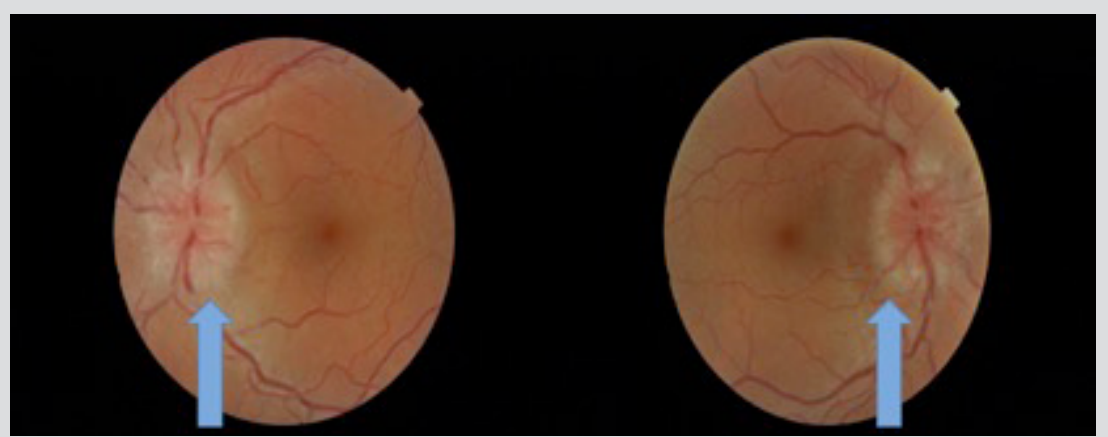

\begin{tabular}{|l|}
\hline \multicolumn{1}{|c|}{ Causes of Papillitis } \\
\hline Multiple Sclerosis \\
\hline Viral Infections \\
\hline Diabetes \\
\hline Giant Cell arteritis \\
\hline Hyperthyroid \\
\hline Syphilis \\
\hline
\end{tabular}

\begin{tabular}{|l|}
\hline \multicolumn{1}{|c|}{ Ocular problems in syphilis: } \\
\hline Blurred vision \\
\hline Reduced acuity \\
\hline Reduced colour perception \\
\hline Interstitial keratitis \\
\hline Uveitis \\
\hline Retinitis \\
\hline Cranial nerve palsy \\
\hline
\end{tabular}

Figure 1. Indistinct optic disc margins but normal CSF opening pressure in papillitis

\section{Table 1. Causes of papillitis}

Table 2. Ocular problems in syphilis infection 


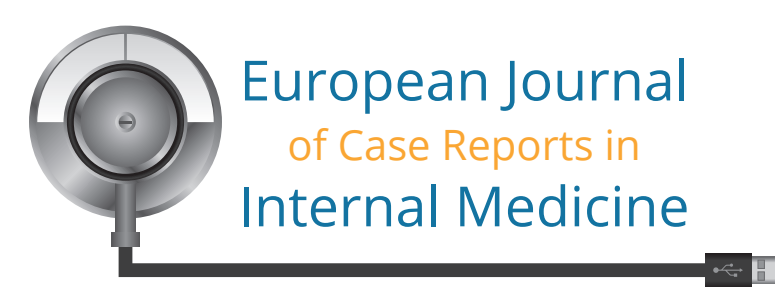

Treatment guidelines have been established and updated ${ }^{[5]}$ and now include the use of either procaine penicillin or benzathine penicillin. The treating physician should be aware of the risk of a Jarish-Herzheimer reaction, a systemic reaction caused by antibacterial lysis of pathogenic cell membranes releasing large quantities of endotoxin into the systemic circulation. It presents as sepsis with hypotension, tachycardia, rash, headache, fever, and vasodilatation. Anti-inflammatory agents (NSAIDS) can be useful prophylaxis while glucocorticoids are not thought to be beneficial.

This patient made a full recovery with resolution of headaches, papillitis, and normalisation of ESR.

\section{REFERENCES}

1. Centres for Disease Control and Prevention. 2015 Sexually Transmitted Diseases Treatment Guidelines - Syphilis. Available from: https://www.cdc.gov/std/tg2015/syphilis. htm [accessed 30/05/2017].

2. Public Health England. Infectious Syphilis and Congenital Syphilis: Recent Epidemiology. Available from: https://www.gov.uk/government/publications/infectious-syphilisand-congenital-syphilis-recent-epidemiologya [accessed 30/05/2017].

3. West of Scotland Sexual Health Managed Clinical Network. West of Scotland Syphilis Protocol. Available from: http://www.sandyford.org/media/3028/west-of-scotlandsyphilis-protocol-ceg-march-2017.pdf [accessed 30/05/2017].

4.> Mehrabian S, Raycheva M, Traykova M, Stankova M, Penev L et al. Neurosyphilis with dementia and bilateral hippocampal atrophy on brain magnetic resonance imaging. BMC Neurol 2012:96 Available from: https://bmcneurol.biomedcentral.com/articles/10.1186/1471-2377-12-96 [accessed 30/05/2017].

5. Kingston M et al. UK National Guidelines on the Management of Syphilis 2015. Int J STD AIDS 2015;0:1-26 (NICE accredited). DOI: 10.1177/0956462415624059 (NICE accredited). 